declared, Kimme Hyrich Speakers bureau: Abbvie unrelated to this study, Grant/ research support from: BMS, UCB, and Pfizer, all unrelated to this study, Anja Strangfeld Paid instructor for: AbbVie, MSD, Roche, BMS, Pfizer, outside the submitted work, Grant/research support from: grants from a consortium of 13 companies (among them AbbVie, BMS, Celltrion, Fresenius Kabi, Lilly, Mylan, Hexal, MSD, Pfizer, Roche, Samsung, Sanofi-Aventis, and UCB) supporting the German RABBIT register, outside the submitted work, Laure Gossec Consultant of: Abbvie, Biogen, Celgene, Janssen, Lilly, Novartis, Pfizer, Sanofi-Aventis, UCB, unrelated to this study, Grant/research support from: Lilly, Mylan, Pfizer, all unrelated to this study, Loreto Carmona: None declared, Elsa Mateus Grant/ research support from: grants from Abbvie, Novartis, Janssen-Cilag, Lilly Portugal, Sanofi, Grünenthal S.A., MSD, Celgene, Medac, Pharmakern, GAfPA; grants and non-financial support from Pfizer, outside the submitted work, Saskia Lawson-Tovey: None declared, Laura Trupin: None declared, Stephanie Rush: None declared, Gabriela Schmajuk: None declared, Patti Katz: None declared, Lindsay Jacobsohn: None declared, Samar Al Emadi: None declared, Leanna Wise: None declared, Emily Gilbert: None declared, Ali Duarte-Garcia: None declared, Maria Valenzuela-Almada: None declared, Tiffany Hsu: None declared, Kristin D'Silva: None declared, Naomi Serling-Boyd: None declared, Philippe Dieudé Consultant of: Boerhinger Ingelheim, Bristol-Myers Squibb, Lilly, Sanofi, Pfizer, Chugai, Roche, Janssen unrelated to this work, Grant/research support from: Bristol-Myers Squibb, Chugaii, Pfizer, unrelated to this work, Elena Nikiphorou: None declared, Vanessa Kronzer: None declared, Namrata Singh: None declared, Manuel F. Ugarte-Gil Grant/research support from: Janssen and Pfizer, Beth Wallace: None declared, Akpabio Akpabio: None declared, Ranjeny Thomas: None declared, Suleman Bhana Consultant of: AbbVie, Horizon, Novartis, and Pfizer (all $<\$ 10,000$ ) unrelated to this work, Wendy Costello: None declared, Rebecca Grainger Speakers bureau: Abbvie, Janssen, Novartis, Pfizer, Cornerstones, Jonathan Hausmann Consultant of: Novartis, Sobi, Biogen, all unrelated to this work $(<\$ 10,000)$, Jean Liew Grant/research support from: Yes, I have received research funding from Pfizer outside the submitted work., Emily Sirotich Grant/research support from: Board Member of the Canadian Arthritis Patient Alliance, a patient run, volunteer based organization whose activities are largely supported by independent grants from pharmaceutical companies, Paul Sufka: None declared, Philip Robinson Speakers bureau: Abbvie, Eli Lilly, Janssen, Novartis, Pfizer and UCB (all $<\$ 10,000$ ), Consultant of: Abbvie, Eli Lilly, Janssen, Novartis, Pfizer and UCB (all $<\$ 10,000$ ), Pedro Machado Speakers bureau: Yes, I have received consulting/speaker's fees from Abbvie, BMS, Celgene, Eli Lilly, Janssen, MSD, Novartis, Pfizer, Roche and UCB, all unrelated to this study (all $<\$ 10,000$ )., Consultant of: Yes, I have received consulting/speaker's fees from Abbvie, BMS, Celgene, Eli Lilly, Janssen, MSD, Novartis, Pfizer, Roche and UCB, all unrelated to this study (all $<\$ 10,000)$., Jinoos Yazdany Consultant of: Eli Lilly and AstraZeneca unrelated to this project DOI: 10.1136/annrheumdis-2021-eular.1632

\section{OP0007 MUC5B PROMOTER VARIANT AND LONG-TERM INCIDENCE OF INTERSTITIAL LUNG DISEASE IN PATIENTS WITH RHEUMATOID ARTHRITIS: A POPULATION BIOBANK STUDY OF 250,000 INDIVIDUALS}

A. Palomäki ${ }^{1}$, T. Laitinen ${ }^{2}$, J. Koskela ${ }^{3}$, A. Palotie ${ }^{3,4,5}$, N. Mars ${ }^{3}$ on behalf of FinnGen. ${ }^{1}$ Turku University Hospital, Centre for Rheumatology and Clinical Immunology, Turku, Finland; ${ }^{2}$ Tampere University Hospital, Administration Center, Tampere, Finland; ${ }^{3}$ University of Helsinki, Institute for Molecular Medicine Finland, FIMM, HiLIFE, Helsinki, Finland; ${ }^{4}$ Massachusetts General Hospital, Analytic and Translational Genetics Unit, Department of Medicine, Boston, United States of America; ${ }^{5}$ Broad Institute of MIT and Harvard, Stanley Center for Psychiatric Research, Cambridge, MA, United States of America

Background: The promoter variant rs35705950 in MUC5B is the strongest known genetic risk factor for rheumatoid arthritis-associated interstitial lung disease (RA-ILD) [1]. There is, however, no large-scale data on the impact of $M U C 5 B$ on the long-term incidence of RA-ILD.

Objectives: To describe long term risk of RA-ILD in RA patients carrying MUC5B variant compared to non-carriers with RA.

Methods: FinnGen is a collection of epidemiological cohorts and hospital biobank samples, linking genotypes with up to 46 years of follow-up within nationwide registries. Diagnoses of RA and ILD were identified from the Finnish national hospital discharge, medication reimbursement and cause-of-death registries. We estimated lifetime risks of ILD by age $80 . M U C 5 B$ is a common variant and has an allele frequency of 0.1 in the Finnish population.

Results: Out of the 248,400 individuals, 5534 patients have been diagnosed with RA, out of whom $178(3.2 \%)$ developed ILD. MUC5B was a strong predictor of ILD in RA patients (HR 2.14,95\%Cl 1.56-2.92). In patients with RA, MUC5B conferred a lifetime risk of $14.5 \%(95 \% \mathrm{Cl} 10.7-18.1 \%)$, compared to $5.2 \%(4.1-$ $6.2 \%$ ) in MUC5B non-carriers with RA (Figure). In the population, MUC5B carriers and $M U C 5 B$ non-carriers had lifetime risks of $3.9 \%$ and $1.3 \%$, respectively.
The risk difference started to emerge at age 65 . The risk was highest in men with RA who are MUC5B carriers: $18.5 \%$ (11.1-25.2\%) developed ILD, compared to $8.5 \%(6.1-10.9 \%)$ of MUC5B non-carriers with RA.

Conclusion: We report findings from a large longitudinal study, showing that MUC5B confers a considerable lifetime risk of RA-ILD, and contributes to increased morbidity. These findings have clinical implications for improving identification of RA patients at high risk of developing ILD.

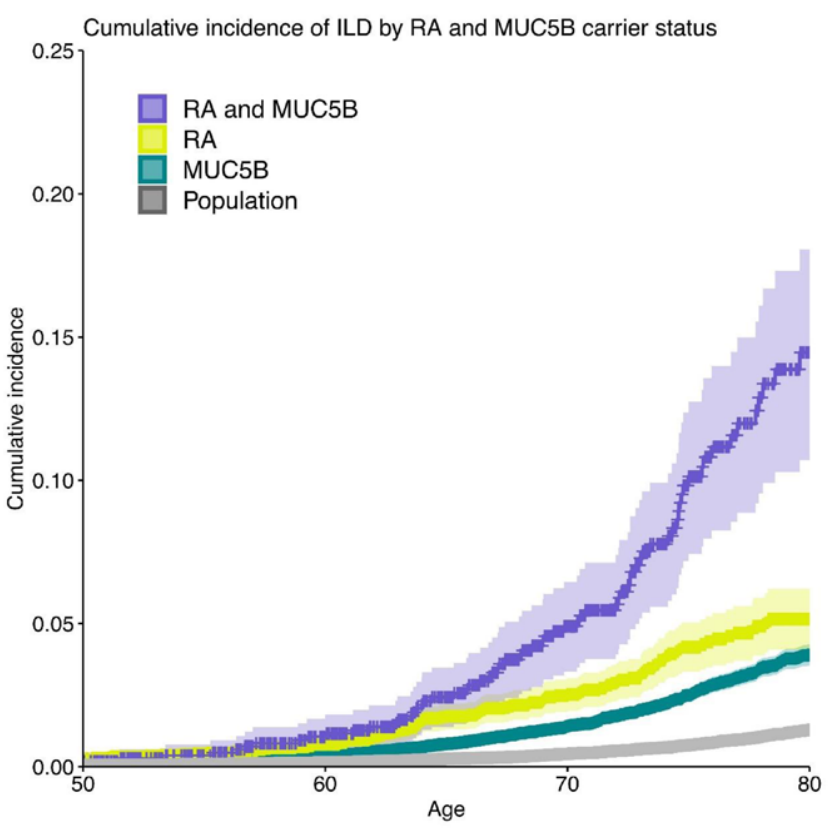

REFERENCES:

[1] Juge P-A, Lee JS, Ebstein E, et al. MUC5B Promoter Variant and Rheumatoid Arthritis with Interstitial Lung Disease. N Engl J Med 2018:379:2209-19 Disclosure of Interests: Antti Palomäki Speakers bureau: MSD, Pfizer, Sanofi, Consultant of: Pfizer, Abbvie, Tarja Laitinen: None declared, Jukka Koskela Speakers bureau: Pfizer, Aarno Palotie: None declared, Nina Mars: None declared

DOI: 10.1136/annrheumdis-2021-eular.619

OP0008

\section{A RANDOMIZED, DOUBLE-BLIND, PLACEBO- CONTROLLED PHASE IIITRIAL OF IVIG $10 \%$ IN PATIENTS WITH DERMATOMYOSITIS. THE PRODERM STUDY: RESULTS ON EFFICACY AND SAFETY}

R. Aggarwal ${ }^{1}$, C. Charles-Schoeman ${ }^{2}$, J. Schess| ${ }^{3}$, Z. Bata-Csorgo ${ }^{4}$, M. Dimachkie ${ }^{5}$, Z. Griger ${ }^{6}$, S. Moiseev ${ }^{7}$, C. V. Oddis ${ }^{8}$, E. Schiopu', J. Vencovský ${ }^{10}$, I. Beckmann ${ }^{11}$, T. Levine ${ }^{12}$, E. Clodi ${ }^{13}$, A. T. Proderm Investigators ${ }^{14}$. ${ }^{1}$ University of Pittsburgh, Rheumatology, Pittsburgh, United States of America; ${ }^{2}$ UCLA Health, Rheumatology, Los Angeles, United States of America; ${ }^{3}$ Friedrich Baur Institute, University of Munich, Neurology, Munich, Germany; ${ }^{4}$ University of Szeged, Faculty of Medicine, Dermatology, Szeged, Hungary; ${ }^{5}$ Univeristy of Kansas, Medical Center, Neurology, Kansas City, United States of America; ${ }^{6}$ University of Debrecen, Department of Internal Medicine, Debrecen, Hungary: ${ }^{7}$ First Moscow State Medical University, Department of Internal Medicine, Moscow, Russian Federation; ${ }^{8}$ University of Pittsburgh, Rheumatology, Piitsburgh, United States of America; ${ }^{9}$ Michigan Medicine, Rheumatology, Ann Arbor, United States of America; ${ }^{10}$ Charles University, Rheumatology, Prague, Czech Republic; ${ }^{11}$ Octapharma PPG, Clinical R\&D, Vienna, Austria; ${ }^{12}$ Phoenix Neurological Associates, Neurology, Phoenix, United States of America: ${ }^{13}$ Octapharma PPG, Global Medical \& Scientific Affairs, Vienna, Austria; ${ }^{14}$ Hospitals in different countries worldwide, RheumatologyDermatology-Neurology, Vienna, Austria

Background: Dermatomyositis (DM) is a rare chronic systemic autoimmune disease with characteristic skin rash and progressive proximal muscle weakness. Current therapies encompass corticosteroids and other immunosuppressants and intravenous immunoglobulins (IVIg), however, none of these therapies are proven by randomized controlled phase 3 studies. There have been no large randomized clinical trials supporting the efficacy and safety of IVIg in DM.

Objectives: The ProDERM study aimed to evaluate the efficacy and safety/tolerability of IVIg in DM patients in a double-blind, randomized, placebo-controlled, international multi-center, phase III clinical trial. 
Methods: The trial consisted of a double-blind, placebo-controlled First Period (16 weeks), in which adult patients with definite or probable DM (according to Bohan and Peter criteria) were randomized 1:1 to either high dose IVIg (2g/kg every 4 weeks) or placebo. Patients on placebo and patients without clinical worsening while on IVIg treatment entered the open label Extension Period (24 weeks) and received $2 \mathrm{~g} / \mathrm{kg}$ IVIg infusions every 4 weeks. To be included, subjects must have active disease with a manual muscle testing-8 (MMT-8) score $<142 / 150$. Patients who showed clinical worsening (defined according to Oddis et al, 2013 - with slight adaptation) at 2 consecutive visits between week 8 and week 16 were switched to the alternate treatment arm.

Primary endpoint was the proportion of responders in the IVIg vs. placebo arm at week 16, where response was defined per 2016 ACR/EULAR Myositis response criteria of at least minimal improvement [Total Improvement Score (TIS) $\geq 20$ points)] and without clinical worsening at 2 consecutive visits up to week 16 .

Results: A total of 95 adult DM patients (mean age: 53 years; $75 \%$ females; $92 \%$ Caucasian) were enrolled, with 47 and 48 randomized to IVIg and placebo, respectively. Baseline clinical characteristics (including medical history and prior DM medication) were balanced between the 2 arms.

The study met the primary endpoint at week 16, with the proportion of responders being significantly higher in the IVIg group (37/47; 78.7\%) as compared to the placebo group (21/48; 43.8\%; p-value 0.0008; Table 1).

Table 1. Total Improvement Score - Analysis of Proportion of Responders at Week 16 (Full Analysis Set, $\mathrm{N}=95$ )

\begin{tabular}{lccc}
\hline TIS Response & $\begin{array}{c}\text { octagam 10\% } \\
\mathrm{N}=\mathbf{4 7}\end{array}$ & $\begin{array}{c}\text { Placebo } \\
\mathrm{N}=\mathbf{4 8}\end{array}$ & $\begin{array}{c}\text { Difference octagam 10\% } \\
\text { - placebo }\end{array}$ \\
\hline $\begin{array}{l}\text { Number (\%) of responders } \\
\text { Difference in response rates }\end{array}$ & $37(78.72 \%)$ & $21(43.75 \%)$ & \\
[95\% Cl] p-value & & 34.97 \\
\hline
\end{tabular}

${ }^{a}$ Cochran-Mantel-Haenszel TestCl=confidence interval; $\mathrm{N}=$ number of patients; TIS=total improvement score

In the analysis of responders per improvement category at Week 16 , a $45.2 \%$ higher response rate for at least moderate improvement (TIS $\geq n 40$ points; $p<$ 0.0001 ) and a $23.6 \%$ higher response rate for at least major improvement (TIS $\geq 060$ points; $p<0.0062$ ) was observed in the IVIG group as compared to the placebo group.

The mean (SD) TIS was significantly higher in IVIg group [48.4 (24.4)] than in placebo arm [21.6 (20.2)] at week 16 (Fig 1).

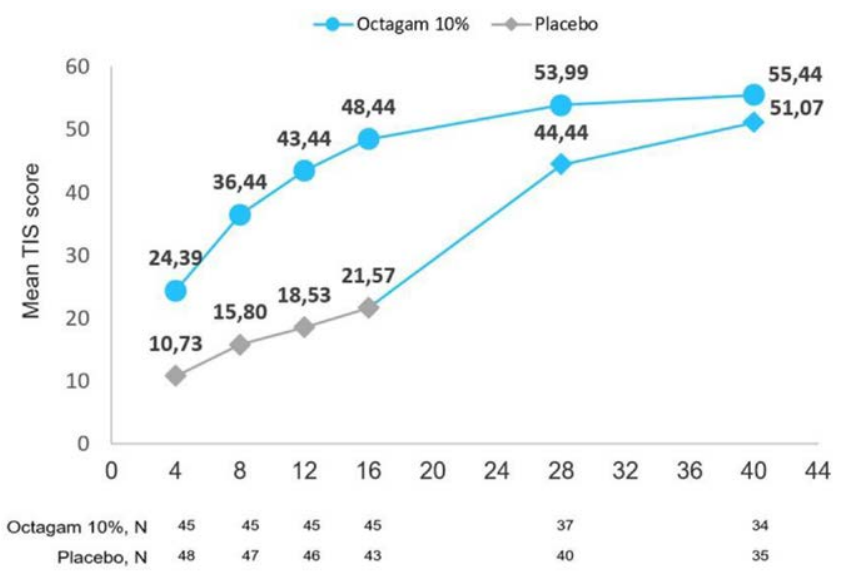

Figure 1.

After switching to IVIG in the Extension Period the placebo group attained a similar response rate at Week 40 as did the IVIg treated patients at Week 16, i.e approx. $70 \%$ for minimal improvement.

In line with the overall primary endpoint, secondary end points including all of the sub-components of TIS except muscle enzyme (MMT-8, MD global, Extramuscular global, patient global, HAQ,) as well as CDASI (Cutaneous Dermatomyositis Disease Area and Severity Index), also showed statistically significant improvement under IVIg treatment compared to placebo treatment.

The safety and tolerability profile of IVIg was consistent with previously reported safety outcomes for IVIg administration.

Conclusion: This is the first large international phase III randomized, placebo-controlled trial demonstrating the efficacy and safety of IVIg as a treatment for patients with DM.

REFERENCES:

[1] Oddis, C. V. et al. Arthritis Rheum (2013), 65, 314-324
Acknowledgements: Acknowledgments to all participating investigators, centers and patients and their families

Disclosure of Interests: Rohit Aggarwal Consultant of: Q32, Alexion, Argenx AstraZeneca, BMS, Boehringer Ingelheim, Corbus, Csl Behring, EMD Serono, Janssen, Kezar, Mallinckrodt, Kyverna, Octapharma, Orphazyme, Pfizer. Grant/research support from: BMS, Mallinckrodt, Pfizer, EMD Serono, Christina Charles-Schoeman Consultant of: Pfizer, Abbvie, Octapharma, Gilead, Regen eron-Sanofi, Grant/research support from: Bristol Myers Squibb, Pfizer, Abbvie, Octapharma, Joachim Schessl Speakers bureau: Octapharma, Grifols, CSL Behring, Consultant of: Octapharma, Zsuzsanna Bata-Csorgo Speakers bureau: Novartis, Sanofi-Genzyme, Ewopharma, Consultant of: Sanofi-Genzyme, Novartis, Ewopharma, Mazen Dimachkie Consultant of: ArgenX, Catalyst, Cello, CSL Behring, EcoR1, Kezar, Momenta, NuFactor, Octapharma, RaPharma/UCB, RMS Medical, Sanofi Genzyme, Shire Takeda, Spark Therapeutics and UCB Biopharma., Grant/research support from: Alexion, Alnylam Pharmaceuticals, Amicus, Biomarin, Bristol-Myers Squibb, Catalyst, Corbus, CSL-Behring, Glax oSmithKline, Genentech, Grifols, Kezar, Mitsubishi Tanabe Pharma, Novartis Octapharma, Orphazyme, Ra Pharma/UCB, Sanofi Genzyme, Sarepta Therapeutics, Shire Takeda, Spark Therapeutics, UCB Biopharma, Viromed/Healixmith., Zoltán Griger Speakers bureau: Abbvie, CSL-Behring, Eli-Lilly, Roche, Boehringer Ingelheim, Consultant of: Octapharma, Sergey Moiseev: None declared, Chester V Oddis Consultant of: EMD Serono; Alexion Pharmaceuticals, Inc, Grant/research support from: Genentech (Clinical trial support); Bristo Myers Squibb (Clinical trial support), Elena Schiopu Consultant of: Octapharma, Grant/research support from: Octapharma, Janssen (Johnson \& Johnson), BMS Pfizer, Abbvie, Jiří Vencovský Speakers bureau: Abbvie, Biogen, MSD, Pfizer, Roche, Sanofi, UCB, Consultant of: Abbvie, Boehringer, Eli Lilly, Octapharma, Gilead, Irene Beckmann Employee of: Octapharma, Todd Levine Shareholder of: Corinthian Reference Labs, CND Life Sciences, Consultant of: Grifols, Octapharma, Alexion, Elisabeth Clodi Employee of: Octapharma PPG, Vienna Austria, and the ProDERM Investigators: None declared DOI: 10.1136/annrheumdis-2021-eular.1389

\section{OP0009-PARE SUCCESSFUL PATIENT EDUCATION ON COVID-19 VACCINE SAFETY IN A LARGE RHEUMATOLOGY COHORT USING INTERACTIVE MOBILE-PHONE VIDEO TECHNOLOGY: CONTEXT, RESULTS, AND NEXT STEPS}

L. Rajagopala ${ }^{1}$, M. Ford ${ }^{1}$, M. Jasim ${ }^{1}$, D. Mulherin ${ }^{1}$, S. Venkatachalam¹, T. Sheeran ${ }^{1}$, J. Bateman ${ }^{1} .{ }^{1}$ The Rheumatology Centre, Cannock Wolverhampton, The Royal Wolverhampton NHS Trust,UK, Rheumatology, Wolverhampton, United Kingdom

Background: There are reported concerns of patient misunderstanding of the COVID-19 pandemic and vaccination safety. It is particularly important that these are understood in patients taking complex immunusuppressive therapies. Rapid delivery of targeted and up-to-date video messages from clinicians sent directly to patients could address patient uncertainty, and improve COVID-19 vaccination uptake. Innovative SMS (short message service) based video message has already shown promise in delivering COVID-19 information to patients [1] We present our experience in creating a tailored vaccine information video sent directly to our large cohort of patients in the UK with a range of rheumatic diseases and report on the learning points going forward.

Objectives: Our three objectives were: (1) educate our patients using an interactive mobile video information resource outlining the safety of the COVID vaccination in these patient groups; (2) better understand our patients' views of the COVID-19 vaccine; (3) evaluate the patient experience of this approach.

Methods: We designed and recorded an 8-minute interactive web-based video delivered education resource designed for mobile phones. This included: aims of the video; details of licenced vaccines; UK vaccination schedule; frequently asked questions; links to national charity resources; our clinician recommendations; a rheumatologist being vaccinated; case studies; and summary data. We produced a simple mobile phone web-based evaluation of the resource, including anonymised patient demographics, their understanding of the safety of the vaccine pre/ post video, and their user experience. Resource and evaluation were piloted by local healthcare professionals, our Patient Participation Group, a national charity, and approved by senior management. We distributed this to our follow-up patient cohort via our hospital SMS provider on 21.12.20, at the start of roll-out of UK vaccines, containing a link to the resource and evaluation.

Results: Of a cohort of 10,981 patients, we had 8886 mobile phone numbers. At Day 14, we recorded 2358 video views (26.3\%) and 664/2358 completed evaluations $(28.1 \%)$. Only one person reported being unable to watch the video. Before watching the video, 348/664 patients $(52.4 \%)$ were unsure if the vaccine was safe and/or recommended for them, rising to $626 / 664$ (94.3\%) post-video. Reasons for uncertainty after the video (38/664) included drug allergy and fertility concerns. Following the video, 509/664 patients $(76.6 \%)$ reported that they were more likely to have the vaccination. The majority of the patients $(614 / 660,93.0 \%)$ 